This is a self-archived author-created version of a paper accepted for publication in the journal 'Minds \& Machines' (Springer). The final publication is available at http://link.springer.com/article/10.1007/s11023-013-9310-x 


\title{
The Logic of Knowledge and the Flow of Information
}

\author{
Simon D'Alfonso
}

\begin{abstract}
In this paper I look at Fred Dretske's account of information and knowledge as developed in Knowledge and The Flow of Information. In particular, I translate Dretske's probabilistic definition of information to a modal logical framework and subsequently use this to explicate the conception of information and its flow which is central to his account, including the notions of channel conditions and relevant alternatives. Some key products of this task are an analysis of the issue of information closure and an investigation into some of the logical properties of Dretske's account of information flow.
\end{abstract}

Keywords Dretske, Information, Knowledge, Closure, Relevant Alternatives

\section{Dretske on Information and Knowledge}

In Knowledge and the Flow of Information Dretske(1981) Dretske develops an account of information and uses it to, amongst other things, construct a theory of knowledge. He uses the Mathematical Theory of Communication (a.k.a Information Theory) as a starting point before acknowledging its limitations and departing to "develop a philosophically useful theory of information" Dretske(1983), p. 55]. After some work he ends up with the following definition:

A signal $r$ carries the information that $s$ is $F=$ The conditional probability of $s$ 's being $F$, given $r$ (and $k$ ), is 1 (but, given $k$ alone, less than 1 )

Here $k$ is a variable that takes into account how what an agent already knows can determine the information carried (for that agent) by a signal 11 Three reasons given by Dretske for stipulating a conditional probability of 1 are:

1. Conjunction principle: if a signal $A$ carries the information that $B$ and the information that $C$, then it also carries the information that $B \& C$. If the conditional probability requirement was relaxed and made lower than 1 then this principle does not hold

2. Xerox principle (transitivity): if $A$ carries the information that $B$, and $B$ carries the information that $C$, then $A$ carries the information that $C$. Once again, if the conditional probability threshold is set to anything less than 1 then this intuitive principle does not hold.

\footnotetext{
${ }^{1}$ Although the $k$ variable which occurs in Dretske's definition relativises information to what the receiver already knows concerning the possibilities at the source, this relativisation is only meant to accommodate the way information is thought about, that the information one can get from a signal depends on what they already know; it does not undermine the essential objectivity of information.
} 
3. If information can be obtained from a signal involving a conditional probability of less than 1, then information loses its 'cognitive punch'. To use an example of Dretske's, think of a bag with ninety four red balls and six white balls. If one is pulled out at random, you cannot know that it was red. Hence why suppose you have the information that it is red?

A main concern against setting the required conditional probability to 1 is that since there are very few conditional probabilities of 1 out there, very little information ever flows. Dretske deals with these concerns by introducing the concepts of fixed channel conditions and relevant alternatives. The basic idea is that the conditional probability requirements are made relative to a set of relevant alternative scenarios, which are determined by a given set of channel conditions. This is not to say that it is impossible for these conditions to fail; only that for the purposes of determining conditional probabilities, given that these conditions do hold, any alternative scenario in which they do not hold can be set aside as irrelevant.

For example, take an actual scenario in which a functioning clock is accurately correlated with the time and is thus transmitting information about the time. Now, there are scenarios alternative to this one, such as cases where the clock has flat batteries, in which the clock's signal is not correlated with the time and it therefore fails to transmit information about the time. If these alternatives are factored into the probability calculations then the probability that it is time $x$ given that the clock shows $x$ is not equal to 1 and consequently the clock would be deemed as not carrying the information that the time is such-and-such according to Dretske's definition.

But the idea is that if the clock is functioning correctly and the right conditions hold (clock calibrated, batteries working, etc), then even though there is technically a non-zero probability that the new batteries could become defective or a mechanism in the clock could break just before someone looks at the clock, such possibilities are excluded in calculating the information that the clock is delivering; such possible (non-actual) but far-flung or improbable alternatives are deemed irrelevant to the information channel 2

After developing his account of information, Dretske uses it to get the following definition of knowledge:

$K$ knows that $s$ is $F=K$ 's belief that $s$ is $F$ is caused (or causally sustained) by the information that $s$ is $F$.

A member of the reliabilist family, this externalist account of knowledge has had a significant presence on the landscape of contemporary epistemology ${ }^{3}$

\subsection{Dretske's Definition and Properties of Information Flow}

It is now time to introduce a symbolic vocabulary for speaking about information flow and list some of the properties which we will consider throughout our investigation. This vocabulary consists of the following:

\footnotetext{
${ }^{2}$ This idea of channel conditions/relevant alternatives will be explored more in Section 3 . There is no determinate method to decide what counts and what does not count as a relevant alternative. In general the selection will depend upon the knowing agent and their environment and will also be a pragmatic decision. Particularly due to this lack of clear determination the notion of relevant alternatives has been a point of philosophical contention and from one point of view its application is seen to be somewhat ad hoc. Nonetheless, it is a valuable idea that can serve as a foundation for accounts that afford a flexible way to realistically talk about information (and knowledge).

${ }^{3}$ A precis of Knowledge and the Flow of Information plus some commentaries can be found in Dretske(1983)].
} 
- Symbols $A, B, C, D, \ldots$ stand for information bearing structures (events / situations / facts / signals).

- The formula $A \sqsupset B$ stands for ' $A$ carries the information that $B$ '.

- $\Rightarrow$ is used to denote a consequence relation that relates such formulas

- In this vocabulary $\sqsupset$ is treated as a meta-connective. The symbols $A, B, C, D, \ldots$ are taken to be statements in the propositional calculus and do not contain $\sqsupset$.

To demonstrate this terminology, take the truism that if $A$ carries the information that $B$, then $A$ carries the information that $B$, which is represented as:

$$
A \sqsupset B \Rightarrow A \sqsupset B
$$

Let it be emphasised that this notation is simply used as a convenient way to express information relations during discussion and is not attached to any particular formal system. If $A$ carries the information that $B$ according to some particular formal system, then $A \sqsupset B$ can be used to express this fact.

The main properties of information flow which we will look at are:

1. Conjunction: $(A \sqsupset B) \wedge(A \sqsupset C) \Rightarrow A \sqsupset(B \wedge C)$

2. Reverse-Conjunction: $A \sqsupset(B \wedge C) \Rightarrow(A \sqsupset B) \wedge(A \sqsupset C)$

3. Disjunction: $(A \sqsupset C) \wedge(B \sqsupset C) \Rightarrow(A \vee B) \sqsupset C$

4. Reverse-Disjunction: $(A \vee B) \sqsupset C \Rightarrow(A \sqsupset C) \wedge(B \sqsupset C)$

5. Transitivity: $(A \sqsupset B) \wedge(B \sqsupset C) \Rightarrow(A \sqsupset C)$

6. Contraposition: $A \sqsupset B \Rightarrow \neg B \sqsupset \neg A$

7. Monotonicity: $A \sqsupset B \Rightarrow A \wedge C \sqsupset B$

Let us take a simpler version of Dretske's definition of information by removing the elements of anti-vacuity and the agent's background knowledge, to get $A$ carries the information that $B$ if and only if $\operatorname{Pr}(B \mid A)=1$. It is this simpler, absolute form of correlation that will generally be used when investigating these properties for accounts of information flow. This will allow us to investigate these properties against the core logic of an account, rather than being affected by the removed extra factors, for some of these principles (e.g. Reverse-Conjunction) would strictly fail to hold using Dretske's full definition (due to anti-vacuity or $k$ ). This stripped down definition of information flow satisfies all of the above conditions 4

\footnotetext{
${ }^{4}$ Technically there are some exceptions involving formulas with a probability of zero and hence undefined calculations. With monotonicity, $\operatorname{Pr}(p \mid p)=1[p \sqsupset p]$ but $\operatorname{Pr}(p \mid p \wedge \neg p)$ is undefined [not $(p \wedge \neg p) \sqsupset p]$. With Reverse Disjunction, $\operatorname{Pr}(p \mid p \vee(q \wedge \neg q))=1[(p \vee(q \wedge \neg q)) \sqsupset p]$ but $\operatorname{Pr}(p \mid q \wedge \neg q)$ is undefined [not $(q \wedge \neg q) \sqsupset p]$.
} 


\section{A Modal Logical Account of Information Flow}

As the cycle of philosophy would have it, Dretske's account has faced its share of criticism. Cohen and Meskin (C\&M) Cohen and Meskin(2006) for example offer a rival theory of information that differs to Dretske's account and explains information in terms of counterfactuals rather than conditional probabilities.5 ${ }^{5}$ Their work takes up on a critique made by Loewer Loewer(1983)], who argues that one problem for Dretske's definition of information is that none of the usual interpretations of probability supports his usage of inverse conditional probability.

Despite these critiques, Dretske's general picture still remains viable. In this section I will show how Dretske's account can simply be translated into a modal logic framework. Let us start with the following 'template' definition of information flow, a definition that Dretske's accords with:

$A$ carries the information that $B$ relative to some system $S$ iff $S$ determines that in every state where $A$ is the case, $B$ is the case

We can formally express this definition simply by using a strict conditional: $\square(A \supset B)$. Thus to begin with we have the following stripped-down definition:

$A$ carries the information that $B={ }_{d f} \square(A \supset B)$

The specific modal logic to be used is the normal modal logic $\mathbf{T}$ (the minimal normal modal logic $\mathbf{K}+$ the $T$ axiom $\square p \supset p$ ), with standard relational semantics being employed Chellas(1980), Priest(2003)]. Thus the condition of reflexivity is imposed upon frames. $A \vdash B$ will be used throughout to express that $A$ is a consequence of or follows from $B$ according to the logic; that is, given $A, B$ can be derived in some suitable proof system for the logic. Clearly there is a correspondence between this modal definition and a conditional probability of 1 . It also stands to reason that since Dretske's account requires a conditional probability of 1 and does not make use of the probability value range $[0,1)$, probability can be replaced with logic. This approach therefore offers one way to deal with Loewer and C\&M's concerns regarding the use of inverse conditional probabilities. The accessibility relation for $\square$ here ranges over possible states, so given the actual state $w$, the set of all states accessed by $w\left(\left\{w^{\prime} \mid w R w^{\prime}\right\}\right)$ can be treated as relevant alternatives to $w$. Given the reflexivity of frames, the actual state is necessarily one of the alternatives that is relevant. This modal logic formulation simply captures the core connection involved in $A$ carrying the information that $B$; every time $A$ occurs, $B$ occurs. The presence of $\square p \supset p$ as a theorem (reflexive frames) ensures veridicality $(A \wedge A \sqsupset B \Rightarrow B){ }^{6}$

\footnotetext{
${ }^{5}$ The account given by C\&M is itself quite problematic, with one reason being that properties of a counterfactual conditional discord too much with properties commonly associated with information flow. If an account of information is to abandon the universality of these properties, it could at least offer some accommodation by delineating the restricted applicability of these properties and account for why they are linked to an ordinary conception of information flow. Demir Demir(2011)] recently pointed a problematic consequence of the counterfactual theory of information that arguably makes it untenable. He shows that given the standard possible worlds account of counterfactuals, according to C\&M's definition " A carries information that B' necessarily implies 'A carries information that B and C' for any $\mathrm{C}$ such that the closest not-C world is more remote than the closest not-B world".

${ }^{6}$ The reflexivity property also gives us other desirable validities which would not hold without it. For example, if $A$ carries the information that $B$ carries the information that $C$, then $A$ and $B$ together carry the information that $C$ :
}

$$
\square(A \supset \square(B \supset C)) \vdash \square((A \wedge B) \supset C)
$$


On top of this base, a couple of additions can be made to reflect certain conditions associated with information flow. As suggested via Dretske's definition, the addition of $\neg \square B$ would be used to express the fact that if $B$ is not a contingent truth, then $A$ does not carry the information that $B$. With $\diamond$ being the dual of $\square(\diamond A \equiv \neg \square \neg A)$, the condition $\diamond A$ can also be added to rule out contradictions trivially carrying information. This gives us the following full definition:

$A$ carries the information that $B=_{d f} \neg \square B \wedge \diamond A \wedge \square(A \supset B)$

As with Dretske's definition, an agent's background knowledge can also easily be incorporated into this definition.

As expected, the core definition $\square(A \supset B)$ validates all of the properties listed in Section 1.1 . As mentioned in Section 1.1, when things like the condition to rule out vacuity $(\neg \square B)$ and the agent's prior information/knowledge are factored in, then technically some of these conditions can fail. For example, Reverse-Conjunction: $\square(A \supset(B \wedge C)) \wedge \neg \square(B \wedge C) \nvdash \square(A \supset C) \wedge \neg \square C$.

\subsection{Variable Relevant Alternatives}

Whilst the validation of the set of test conditions given in Section 1.1 is essentially correct, there are cases where it seems reasonable to talk of information flow in a way that violates one or more of the conditions. For example,

violations of transitivity occur when the signal type $A$ of the first channel is inconsistent with the background conditions on the channel from $B$ to $C$. For example, let $A$ be 'the doorbell is short circuited', $B$ be 'the doorbell is ringing', and $C$ be 'someone is at the door'; that $B$ carries the information $C$ is one of Dretske's standard examples. $\ldots$ it seems intuitively reasonable to accept that $A$ carries the information $B, B$ carries the information $C$, but $A$ does not carry the information $C$. Thus transitivity should be rejected by any theory of information flow. Wobcke(2000), p. 105]

The task here now is to devise a system that accommodates the form of transitivity embodied in the Xerox principle whilst rejecting transitivity in general. Let us begin by laying out this example:

1. the doorbell is short circuited carries the information that the doorbell is ringing

2. the doorbell is ringing carries the information that someone is at the door

3. the doorbell is short circuited does not carry the information that someone is at the door

What is going on here is that strictly speaking relation 2 only holds if the doorbell is not short circuited. If the doorbell is ringing because it is short circuited, then it plainly does not carry the information that someone is at the door. So with the possibility that the doorbell is short circuited in such a scenario, the doorbell ringing does not carry the information that someone is at the door. Given this, one option is to deny relation 2 .

But there still seems to be a sense in which we want to maintain the validity of both 1 and 2 , in which case there is a failure of transitivity. How does this work? Well, if relation 2 above 
holds, it will involve the exclusion of all alternatives in which the doorbell is short-circuited. So there are alternatives associated with relation 1 that will not be associated with relation 2 . Thus both relation 1 and relation 2 hold relative to a certain set of alternatives. Since the set of accessed alternatives for a state is captured using $\square$, the employment of a multi-modal logic will easily allow us to capture this idea. So now let $\square_{1}, \square_{2}, \ldots, \square_{n}$ stand for a group of operators, each one with its own accessibility relation $R_{1}, R_{2}, \ldots, R_{n}$. A relation such as $w R_{1} w^{\prime}$ means that $w^{\prime}$ is an accessed alternative for $\square_{1}$. This can be used to express the idea that according to the context or channel conditions associated with $\square_{1}, w^{\prime}$ is a relevant alternative.

We can now capture both the Xerox principle and the rejection of transitivity. The Xerox principle applies when the set of relevant alternatives is fixed:

$$
\square_{x}(A \supset B) \wedge \square_{x}(B \supset C) \vdash \square_{x}(A \supset C) \text { (Xerox Principle) }
$$

On the other hand, if relevant alternatives vary across information statements then transitivity need not hold:

$$
\square_{x}(A \supset B) \wedge \square_{y}(B \supset C) \nvdash \square_{z}(A \supset C) \text { (Non-Transitivity) }
$$

The addition of $\square_{x} A \supset \square_{y} A$ as an axiom can be used to define a frame condition which ensures that the alternatives for $\square_{y}$ form a subset of the alternatives for $\square_{x}\left(w_{1} R_{y} w_{2} \Rightarrow w_{1} R_{x} w_{2}\right)$.

Let us continue by applying this idea to the following standard example. Take the following predicates:

- $P a=a$ is a penguin

- $B a=a$ is a bird

- $F a=a$ flies

and the following three information relations:

1. $P a \sqsupset B a$

2. $B a \sqsupset F a$

3. $F a \sqsupset \neg P a$

Relations 1 and 3 are zoological truths that are true in any state of the world. Translating them to a relevant alternatives system and the modal logic framework, we can assign them the same accessibility relation to get:

- $\square_{1}(P a \supset B a)[1]$

- $\square_{1}(F a \supset \neg P a)[3]$ 
The states associated with relation 2 exclude flightless birds and form a subset of the states associated with 1 and 3 . In order to capture this in the modal logic framework we include $\square_{1} A \supset$ $\square_{2} A$ as an axiom and represent relation 2 with:

- $\square_{2}(B a \supset F a)[2]$

Let $\Gamma$ stand for a collection of premises consisting of these three formulas representing the information relations. So our setup here involves the multi-modal logic per the outline above with operators $\square_{1}$ and $\square_{2}$, the axiom $\square_{1} A \supset \square_{2} A$ connecting them and $\Gamma$ as the set of premises.

As it is this setup has some clearly unacceptable consequences, to the point of triviality:

- $\Gamma, P a \vdash \neg P a$

- $\Gamma \vdash \square_{2}(P a \supset \neg P a)[a$ being a penguin carries the information that $a$ is not a penguin $]$

What has gone wrong here is a lack of required qualifications. If $\square_{2}(B a \supset F a)$ is to hold, then it must not be the case that $\square_{2}$ ranges over alternatives in which $P a$ is the case; $\square_{2}(B a \supset F a)$ holds or fails to hold relative to certain conditions. If $a$ being a bird carries the information that $a$ flies, then $P a$ is not amongst the relevant alternatives being considered. This can be formally expressed as:

$$
\square_{2}(B a \supset F a) \supset \square_{2} \neg P a^{8}
$$

With all this in place, relation 2 becomes:

- $\square_{2}(B a \supset F a) \supset \square_{2} \neg P a\left[2^{\prime}\right]$

Letting $\Gamma$ now stand for the collection consisting of formulas 1, 3 and 2', we have:

- $\Gamma, P a \vdash \neg \square_{2}(B a \supset F a)$

- $\Gamma, P a \nvdash \neg P a$

- $\Gamma \nvdash \square_{2}(P a \supset \neg P a)$

If $\square_{2}(B a \supset F a)$ is given, then we get the following two validities:

- $\Gamma, \square_{2}(B a \supset F a) \vdash \square_{2} \neg P a$

- $\Gamma, \square_{2}(B a \supset F a) \vdash \square_{2}(P a \supset \neg P a)$

\footnotetext{
${ }^{7}$ Other clearly problematic results follow from further additions. For example, adding the zoological truth $\square_{1}(P a \supset$ $\neg F a$ ) leads to the problematic $\square_{2}(P a \supset \neg B a)$.

${ }^{8}$ Perhaps expressed more clearly with the equivalent: $\diamond_{2} P a \supset \square_{2}(B a \supset F a)$
} 
Whilst the second of these might seem problematic, with an added requirement that the antecedent of an information relation be possible, $P a$ would fail to carry the information that $\neg P a$ since $P a$ is not possible.

Monotonicity failure is similarly explained. From $\square_{2}(B a \supset F a)$ and the statement $\square_{2}(B a \supset$ $F a) \supset \square_{2} \neg P a$ it follows that $\neg \searrow_{2} P a$. Given a definition of information with a qualification ruling out impossible signals, this means that $(B a \wedge P a) \sqsupset F a$ would not hold 9 For contraposition, $\square_{2}(\neg F a \supset \neg B a)$ rightly follows from $\square_{2}(B a \supset F a)$. But $\square_{1}(\neg F a \supset \neg B a)$ does not follow from $\square_{2}(B a \supset F a)$, which is as it should be.

This multi-modal framework provides a simple but effective way to talk about information flow, in which we can easily make use of the proof procedures available for modal logic to explore valid information relations. As we have seen, it requires that the conditions which preclude the presence of an information relation from $A$ to $B$ be spelled out. Statements of information carriage are of the form: $\square(A \supset B) \supset \square \neg\left(C_{1} \vee C_{2} \vee \ldots \vee C_{n}\right)$. In Dretskean parlance, $\neg C_{1}, \neg C_{2}, \ldots, \neg C_{n}$ can be described as channel conditions. In this way, since $\neg C_{1}, \neg C_{2}, \ldots, \neg C_{n}$ hold over the given set of relevant alternatives, information-carrying signals cannot tell us anything new about these channel conditions relative to this set. Whilst theoretically or philosophically the list of conditions can be open-ended and indefinite, in practical applications with a finite, manageable list of predicates, an appropriate listing of conditions will be possible 10

\section{Information Closure}

One point of contention in epistemology is the Epistemic Closure Principle Luper(2010), Collins(2006)], according to which the set of things one knows is closed under entailment. There are many ways to formulate the closure principle. For our purposes, we will settle on the following broad formulation:

If $S$ knows that $p$ and knows that $p$ implies $q$, then $S$ is in a position to know that $q$. $\operatorname{Steup}(2005)$. p. 2]

In this section, our focal point will be the following informational versions of the closure principle:

- If $S$ holds the information that $p$ and $S$ holds the information that $p$ implies $q$, then $S$ holds the information that $q$.

- If $S$ holds the information that $p$ and $p$ carries the information that $q$, then $S$ holds the information that $q$.

The main task of this section is to analyse Dretske's rejection of closure for information and knowledge and begin teasing out the logic of his account. Although the foundations for this rejection preceded his informational account of knowledge, if closure for knowledge is to fail in this account, then closure for information must also fail. Problematically for Dretske though, given the definition

\footnotetext{
${ }^{9}$ Regarding $\square_{1}, \square_{1}(B a \supset F a)$ is illegitimate and leads to the incorrect $\square_{1}(P a \supset \neg P a) . \square_{1}(B a \wedge P a \supset \neg F a)$ is derivable in the system so the addition of $\square_{1}(B a \wedge P a \supset F a)$ would lead to the unacceptable $\neg \nabla_{1}(B a \wedge P a) \vee \diamond_{1}(F a \wedge$ $\neg F a)$. Hence $\square_{1}(B a \wedge P a \supset F a)$ is also illegitimate.

${ }^{10}$ Perhaps in less practical discourse, a catch-all clause $C$ could act as a stand-in of sorts, such that $\square(A \supset B) \supset$ $\square \neg C$.
} 
he uses in his account of information, it seems like closure for information does hold. I will show that this issue rests on a limitation with the technical machinery of Dretske's definition before showing how the failure of closure for information he has in mind can be simply captured using the multi-modal framework given in the previous section.

There is a variety of reasons why the closure principle is rejected ${ }^{11}$ Whilst the principle does seem quite sensible and explanatorily valuable, it is not without good reason that some epistemologists such as Dretske and Nozick deny it. Here we are interested in Dretske's argument from his relevant alternatives analysis of knowledge. For Dretske, one can know that $p$ because they have ruled out all relevant alternatives to $p$ and even if they know that $p \rightarrow q^{12}$, since they cannot rule out all the alternatives relevant to $q$ (some of which will be irrelevant to $p$ ) they are not in a position to know $q$.

The idea that closure is not universally valid because of this analysis of knowledge was suggested early by Dretske [Dretske(1970), Dretske(1971)] and originally formulated in terms of conclusive reasons. Basically, the idea is that $R$ is a conclusive reason for $p$ if it is the case that within all the relevant alternatives for $p$, if not- $p$ holds then $R$ does not hold. Given that knowledge requires conclusive reasons, sometimes knowledge will not be closed under known implication. Take the following classic example from Dretske(1970)].

You are at the zoo and go to the zebra section. Your visual experience of a zebra is a conclusive reason for, and so provides you with the knowledge that the animal before you is a zebra. The zebra visual experience is a conclusive reason because in all the relevant alternatives ${ }^{13}$ where it is false that a zebra is before you in the enclosure there is no zebra-like visual experience. Suppose that this set of relevant alternatives consists of worlds with standard zoo scenarios in which other animals such as tigers or camels are in the enclosure.

Now, something being a zebra implies that it is not a cleverly disguised mule painted to look like a zebra. This is a necessary truth that you know. Does it therefore follow that you know that the animal before you is not a cleverly painted mule? According to the conclusive reasons analysis, the answer is no. This is because you do not have a conclusive reason for it not being the case that the animal is a disguised mule. To have a conclusive reason, you would need to be able to rule out relevant alternatives where the animal is a disguised mule. But since your visual experience would be the same for a zebra and a disguised mule, it is not the case that if the animal was a painted mule, then you would not have the zebra-like visual experience. That is, the visual experience is not a conclusive reason for not-mule.

The catch here is that "the negation of a proposition $p$ is automatically a relevant alternative to $p$ (no matter how bizarre or remote not- $p$ might be) but often not a relevant alternative to things that imply $p$. ... [so] we can know something $p$ only if we can rule out not- $p$ but we can know things that entail $p$ even if we cannot rule out not- $p[\operatorname{Luper(2010)]}$. Thus one can know that zebra (ordinary proposition), without knowing that not-mule (the equivalent of a skeptical proposition or what Dretske terms 'heavyweight' proposition).

By 1981 with Knowledge and the Flow of Information Dretske framed all of this in terms of his information-theoretic account of knowledge. With this account, another way to look at all of this is that the zebra visual signal carries the information that the creature is a zebra given that not-mule is a channel condition. When not-mule is a channel condition, the signal cannot also carry the information that not-mule.

\footnotetext{
${ }^{11}$ See $\operatorname{Luper(2010)}$ for an overview.

$12 \rightarrow$ is being used here as a general representation of an implication/conditional

${ }^{13}$ This is sometimes talked about in terms of nearby possible worlds
} 
Since Dretske bases his definition of knowledge on information, it should follow that information is also not closed under entailment. Relatively recently there has been some new discussion in the literature regarding the matter, with Jager [Jager(2004)] suggesting that given Dretske's definition of information, information, hence knowledge, are closed under known entailment.

Jager's technical argument does not really trouble Dretske's account and Dretske has no problems himself providing a defence [Dretske(2006)] on similar terms to my own response. But, as I will show, ultimately some clarifications and minor modifications to Dretske's formalisation of his account are required. Let us start with Jager's argument, which shows that information closure follows from Dretske's probabilistic definition of information.

Jager approaches the issue by examining whether information is closed under known entailment using the following formulation:

PIC : If $r$ carries, relative to the subject $K$, the information that $p$, and $K$ knows that $p$ entails $q$, then $r$ carries, relative to $K$, the information that $q$.Jager(2004), p. 192]

Let $e$ stand for some appropriate ordinary proposition (such as 'zebra'), $h$ stand for some skeptical/heavyweight proposition (such as 'disguised mule') and $k$ stand for the agent's background knowledge. According to Dretske's definition of information, the veridicality of knowledge and the probability calculus, if $r$ carries the information that $e$ we have:

(1) $\operatorname{Pr}(e \mid r \wedge k)=1 \& \operatorname{Pr}(e \mid k)<1$

which coupled with the entailment:

(2) $\vdash e \rightarrow \neg h$

results in:

(3) $\operatorname{Pr}(\neg h \mid r \wedge k)=1$

In keeping with Dretske's condition that the prior probability of the proposition in question is less than one, it only remains to be shown that $\operatorname{Pr}(\neg h \mid k)<1$. Jager does this by simply noting that since $h$ is a skeptical hypothesis, we are not entitled to be certain that it is false; there is at least a minute, non-zero probability that it is true. Therefore $\operatorname{Pr}(\neg h \mid k)<1$.

Given all of this, information is apparently closed according to Dretske's definition. Shackel Shackel(2006)] and Baumann [Baumann(2006)] offer some manoeuvres which would provide a way out of Jager's problem. But whilst they attempt to defend Dretske, their defences are not in line with Dretske's overall picture and ultimately do not seem to be very helpful ${ }^{14}$ The issue they are looking to resolve has not been formulated in the right way. Even though it is the case that the signal $r$ need not carry the information that $\neg h$, according to their arguments this is because the agent in question already possess that information. But Dretske is going to want a different form of

\footnotetext{
${ }^{14}$ This is further evidenced by a subsequent case that Shackel makes, according to which, Dretske is committed to closure for a modified version of closure, a general closure principle for signalled information.
} 
closure failure, according to which one can be informed that $e$ and informed that $e \rightarrow \neg h$ without at all being in a position to be informed that $\neg h$ due to the signal $r$ not carrying this information at all because of the relevant alternatives analysis (not just that the signal does not inform the agent that $\neg h$ because they already have this information).

If the motivations and foundations for Dretske's account are accepted (which of course, they need not be), then Dretske's response to this debate is successful and shows why the contributions from Jager, Shackel and Baumann miss the mark. But whilst Dretske is able to defend his scheme, his formal definition of information flow is not powerful enough to adequately serve it. Ultimately, his probability definition is not enough to capture the rich idea of relevant alternatives. So rather than upheaving things and developing "a theory of perceptual information that differs considerably from the one Dretske proposes" [Jager(2004), p. 195], what is needed is a replacement of Dretske's formal apparatus with something such as the multi-modal framework, which can adequately do the job.

A consequence of this analysis is that at some level transitivity must fail. As was mentioned earlier, Dretske endorses the Xerox principle. This is the only reference he makes to transitivity and would seem to imply that transitivity universally holds. But as has been established, there is a difference between the Xerox principle and transitivity in general, with the former being a special case of the latter. With this point, we can associate the failure of information closure with the failure of information transitivity.

Let us investigate all of this by returning to the zebra example, beginning with the following proposition assignments:

- $v=$ zebra visual signal

- $z=$ zebra

- $m=$ painted disguised mule

Letting $\mathrm{I}_{a} p$ stand for 'agent $a$ is informed that $p$ ' in some logic of being informed, we can represent the failure of information closure in the zebra scenario as:

$$
\mathrm{I}_{a} z \wedge \mathrm{I}_{a}(z \rightarrow \neg m) \nvdash \mathrm{I}_{a} \neg m
$$

This invalidity goes hand in hand with the following invalidity in a logic for information flow:

$$
(v \sqsupset z) \wedge(z \sqsupset \neg m) \nRightarrow v \sqsupset \neg m
$$

In the zebra scenario the zebra visual signal one receives carries the information that the creature before them is a zebra. Something being a zebra carries the information that it is not a mule painted to resemble a zebra. Does it follow from this that the visual signal they are receiving carries the information that the creature before them is not a mule painted to resemble a zebra? Dretske would want to say no; in effect he would have to deny the transitivity of information flow in this case. Otherwise if $v \sqsupset \neg m$ were to hold the agent could come to know $\neg m$ by receiving the information that $\neg m$ via $v$ and forming the appropriate belief. But with Dretske's probabilistic definition of information, we have: 
- $\operatorname{Pr}(z \mid v)=1$

- $\operatorname{Pr}(\neg m \mid z)=1$

- $\operatorname{Pr}(\neg m \mid v)=115$

So a different formal apparatus is required. Applying the multi-modal logic framework, we start with the following:

- $\square_{x}(v \supset z)$

- $\square_{y}(z \supset \neg m)$

- $\square_{z}(v \supset \neg m)$

Clearly transitivity is no longer inevitable:

$$
\square_{x}(v \supset z) \wedge \square_{y}(z \supset \neg m) \nvdash \square_{z}(v \supset \neg m) .
$$

How $x, y$ and $z$ are assigned depends on one's approach to setting relevant alternatives. A basic distinction to make is between relevant alternative accounts such as Dretske's that deny closure and contextualist relevant alternative accounts that accept closure Black(2006).

For Dretske, the set of relevant alternatives depends on the proposition in question. $\square_{x}(v \supset z)$ is not going to range over irrelevant alternatives with the non-actual possibility that the zoo has a painted mule. $\square_{y}(z \supset \neg m)$ will presumably be able to range over all alternatives, since it is a necessary truth. $\square_{z}(v \supset \neg m)$ will once again range over another set of alternatives, ones where the possibility of a mule being in the zoo's enclosure are counted. Whilst 'mule' is not relevant to 'zebra', it is relevant to 'not-mule'. So according to this Dretskean picture, we have the following:

- $\vdash \square_{y} A \supset \square_{x} A$

- $\vdash \square_{y} A \supset \square_{z} A$

- $\square_{x}(v \supset z) \wedge \square_{y}(z \supset \neg m) \nvdash \square_{z}(v \supset \neg m)$

Since $x \neq z$, the information flow is not transitive and $v$ does not carry the information that $\neg m$. $\square_{x}(v \supset \neg m)$ is derivable, but since $\neg m$ is a channel condition relative to the set indexed by $x$, this

\footnotetext{
${ }^{15} \mathrm{On}$ a related note, another part of Dretske's account which would need to be revised is information nesting as mentioned in Section 1

$\ldots$ if a signal carries the information that $s$ is $F$, it also carries all the information nested in $s$ 's being $F$. $\cdots$

The information that $t$ is $G$ is nested in $s$ 's being $F=s$ 's being $F$ carries the information that $t$ is $G$.

$$
\text { Dretske(1981), p. 71] }
$$

He also distinguishes between information that is analytically nested and information that is nomically nested. As the zebra-mule example shows, caution must be exercised with nesting and its applicability regulated, for although 'not-mule' is necessarily implied by 'zebra', the information that 'not-mule' is not necessarily analytically nested in the information that 'zebra'.
} 
does not represent an information relation in Dretske's account. One benefit of such closure denial is that it affords a way to counter the skeptic and maintain knowledge of ordinary propositions whilst accommodating the fact that we do not know skeptical heavyweight propositions. One issue though concerns the legitimacy of such shifting across sets of relevant alternatives.

Contextualists such as Stine [Stine(1976)], who accuse Dretske of cross-context equivocation, prefer another way around the skeptic ${ }^{16}$ They posit an interpretation of the relevant alternatives idea whereby each of the premises involved in the evaluation of a knowledge statement (or information in our case) shares the same set of relevant alternatives. What determines which set of relevant alternatives is used is not "certain features of the putative subject of knowledge (his/her evidence, history, other beliefs, etc.) or his/her objective situation (what is true/false, which alternatives to what is believed are likely to obtain, etc.), but rather features of the knowledge attributor(s)' psychology and/or conversational-practical situation" Rysiew(2011).

This dominant form of contextualism, 'attributor contextualism', means that in ordinary discourse the claim that one knows the animal is a zebra (based on $v \sqsupset z$ ) and the claim that one knows it is not a disguised mule are both true. In a skeptical discourse on the other hand, both claims are false. Without any change in the subject's situation, change in the attributor's conversational-practical situation means that the subject goes from knowing to not knowing.

In terms of the modal logical analysis, let 1 and 2 index two different contexts or sets of relevant alternatives, such that the set indexed by 1 (lower standard) is a proper subset of that indexed by 2 (higher standard) [axiom $\square_{2} A \supset \square_{1} A$ ]. The argument to be evaluated is of the following form:

$$
\text { - } \square_{1}(v \supset z), \square_{2}(z \supset \neg m) \vdash \square_{x}(v \supset \neg m)
$$

Evaluating against the lower standards context, $x$ is set to 1 and $\square_{1}(v \supset \neg m)$ is derivable. Evaluating against the higher standards context, $x$ is set to 2 and $\square_{2}(v \supset \neg m)$ is not derivable. In this way closure is preserved.

This is a fairly crude outline of the contextualist approach, but it will serve as a reference point to further discuss the Dretskean position and motivation for legitimating shifts in relevant alternatives. Dretske adopts what he terms 'modest contextualism', whereby the set of relevant alternatives for a proposition is determined by certain objective features of the putative knower and their environment, not subjectively by the attributor 17 Unlike 'radical contextualism', the attributor's conversational-practical situation should not 'rob' subjects of knowledge. It is this feature of contextualism that Dretske finds disagreeable [Dretske(2004), Dretske(2005)]: if someone can be robbed of their knowledge simply by the contemplation of skeptical scenarios then something is amiss.

So if one wants to defeat the skeptic and maintain knowledge of ordinary propositions whilst acknowledging that the negation of skeptical/heavyweight propositions are not known, then a strategy employing shifts across sets of relevant alternatives is one way to go. By denying closure in this way, if it is true that a subject knows an ordinary proposition based on the right information given their situation, this knowledge claim stays true; it does not have to be given up so easily.

In closing this section it is important to emphasise that the Dretskean rejection of closure only applies to certain cases; it is certainly not the case that this position is anti-closure. We can gen-

\footnotetext{
${ }^{16}$ Heller Heller(1999)] offers a good explication of Dretske's type of relevant alternatives account that answers opponent contextualists such as Stine.

${ }^{17}$ As suggested by an anonymous referee, perhaps a better name for Dretske's view would be 'situationism', since it relies only on objective features of the epistemic agent's situation and not on contexts of discourse and such.
} 
erally still gain information/knowledge of some ordinary proposition $B$ by inferring it from the information/knowledge of some $A$. For example, replace 'mule' with 'tiger' and you get information transitivity and knowledge closure in the zoo scenario. So in the majority of ordinary cases information/knowledge will be preserved under entailment. Indeed,

some of these reactions [to closure denial] are, I think, a bit overdone. To deny closure is not to say that you can never know (find out, discover, learn) that $Q$ is true by inferring it from a $P$ that you know to be true. It is merely to deny that this can be done for any $Q .[$ Dretske(2005), p. 17]

\section{Variable Relevant Alternatives and the Other Information Flow Properties}

In this section we consider how the conjunction and disjunction principles fare under a Dretskean relevant alternatives account.

Simply due to their structure, the Reverse-Conjunction and Reverse-Disjunction principles have less room to be affected:

- Reverse-Conjunction: - $\square_{x}(A \supset(B \wedge C)) \vdash \square_{y}(A \supset B) \wedge \square_{z}(A \supset C)$

- Reverse-Disjunction: - $\square_{x}((A \vee B) \supset C) \vdash \square_{y}(A \supset C) \wedge \square_{z}(B \supset C)$

Given that there is only one index $x$ in the antecedent and that the statements within the scope of $\square_{x}$ entail the formulas within the scopes of $\square_{y}$ and $\square_{z}$, for either of these two principles I see no legitimate way to set $x, y$ and $z$ such that they would fail.

There is one point related to the Reverse-Conjunction principle that is worth mentioning. Although Kripke's 'red barn' example ${ }^{18}$ was intended as a counterexample to Nozick's tracking theory of knowledge, it might seem like such examples could pose a problem for Dretske's account. In fact, as pointed out by Adams [Adams(2005), p. 34], Lehrer [Lehrer(1990), p. 184] suggests that such cases are problematic for externalist accounts such as Dretske's ${ }^{19}$ In the 'red barn' example Bob is travelling through fake barn county, in which real barns and fake barns are distributed. Since for some reason it is not possible to fake a red barn all the red barns are real. If Bob sees a red barn what information does he receive?

Since in all relevant alternatives every time something looks like a red barn $(L r b)$ it is a red barn, Bob receives the information that the object is a red barn $(B o \wedge R o)$. Does it follow from this that he receives the information that the object is a barn: $B o$ ? Some might say that since there are relevant alternatives in which something looks like a barn $(L b)$ but is not a barn, then Bob is not receiving the information that the object is a barn. Given Dretske's information-based definition of knowledge, this would lead to the problematic conclusion that Bob can know that $B o \wedge R o$ but not know that $B o$. The problem with this purported counterexample is that it switches from red

\footnotetext{
${ }^{18}$ Kripke presented this and some other examples at an APA session in the early 1980s. An extensive published critique of Nozick's account, including discussion of the red barn example, can be found in Kripke(2011).

${ }^{19}$ Dretske Dretske(2003), p. 117] briefly discusses this example and offers the case for why it does not affect his account, even if it affects Nozick's. Adams Adams(2005), p. 8] offers the case for why Kripke's example does not undermine Nozick's or Dretske's account either.
} 
barn to just barn when considering what visual signal Bob receives, when it should just be fixed on red barn. The information that $B o$ should be the judged by the signal $L r b$, not the signal $L b$ : $L r b \sqsupset(R o \wedge B o),(R o \wedge B o) \sqsupset B o \Rightarrow L r b \sqsupset B o$.

The Conjunction and Disjunction principles are more interesting:

- Conjunction: - $\square_{x}(A \supset B) \wedge \square_{y}(A \supset C) \vdash \square_{z}(A \supset(B \wedge C))$

- Disjunction: - $\square_{x}(A \supset C) \wedge \square_{y}(B \supset C) \vdash \square_{z}((A \vee B) \supset C)$

Is it possible to come up with actual examples of information flow failing the Conjunction or Disjunction principles under Dretske's account? To begin with, let us first consider another principle:

$$
\text { Double Conjunction: - }(A \sqsupset C) \wedge(B \sqsupset D) \Rightarrow(A \wedge B) \sqsupset(C \wedge D)
$$

One thing to note about this principle is that even if the Conjunction principle were to hold, then the failure of this principle would mean that information-based knowledge is not closed under conjunction: $\mathrm{K} c \wedge \mathrm{K} d \nvdash \mathrm{K}(c \wedge d)$. For it could be the case that one comes to know $C$ via $A$ and comes to know $D$ via $B$ without receiving a signal that carries the information that $C \wedge D$. However, rather than focusing on the Double Conjunction principle in particular, I will now cut straight to the Conjunction principle, showing how it, and a fortiori the Double Conjunction principle, can fail.

Returning to the zebra scenario, take the following two information statements:

- $v \sqsupset z$

- $v \sqsupset z \vee m$

In this example, $v \sqsupset z$ is as usual. $v \sqsupset z \vee m$ is made relative to a wider range of relevant alternatives, ones in which all animals, including mules are considered. With regards to the standard zoo scenario I do not see a problem in asserting both $v \sqsupset z$ and $v \sqsupset z \vee m$ under Dretske's account. Translating these information statements to the modal logic framework we have:

- $\square_{x}(v \supset z)$

- $\square_{y}(v \supset z \vee m)$

- the axiom $\square_{y} A \supset \square_{x} A$

From here it is legitimate to add

- $\square_{y}(z \supset \neg m)$

- $\square_{y}(m \supset \neg z)$ 
from which can be derived

- $\square_{y}(v \supset((z \wedge \neg m) \vee(m \wedge \neg z)))$

Given this deduction, we now have the following two information statements:

- $v \sqsupset z$

- $v \sqsupset((z \wedge \neg m) \vee(m \wedge \neg z))$

Can these two information statements be joined as per the Conjunction principle to get $v \sqsupset$ $(z \wedge((z \wedge \neg m) \vee(m \wedge \neg z)))$ ? Suppose that there were a suitable set of relevant alternatives indexed by $z$ against which this information statement were judged: $\square_{z}(v \supset(z \wedge((z \wedge \neg m) \vee(m \wedge \neg z))))$. $\square_{z}(v \supset(z \wedge \neg m))$ is a consequence of this, from which it follows that $\square_{z}(v \supset \neg m)$ is also a consequence. But this would then mean that $v \sqsupset \neg m$, something that Dretske does not want.

Let $\dashv$ here stand for logical equivalence. In general, for any account of $\sqsupset$ which has the following, such a consequence will follow:

1. $B \dashv \vdash C, A \sqsupset B \Rightarrow A \sqsupset C$

2. $A \sqsupset(B \wedge C) \Rightarrow(A \sqsupset B) \wedge(A \sqsupset C)$

Rather than abandon either of these properties, it is the Conjunction principle that should go. The basic diagnosis is that the statements $v \sqsupset z$ and $v \sqsupset((z \wedge \neg m) \vee(m \wedge \neg z))$ are made relative to different sets of relevant alternatives in such a way that they cannot be coherently combined. Thus as it turns out Dretske cannot have an absolute Conjunction principle.

This example feeds into a similar example that counters the Double Conjunction Principle. Let $e$ stand for a statement asserting that the animal in question is an equid. Even if for some reason $v \sqsupset((z \wedge \neg m) \vee(m \wedge \neg z))$ was denied, the statement $e \sqsupset((z \wedge \neg m) \vee(m \wedge \neg z))$ is indisputable ${ }^{20}$

Suppose that the zoo goer was told by a reliable zoo keeper that the animal in the enclosure is an equid. They look at the animal and see that it is a zebra. Whilst they have the signals $v$ and $e$, they do not have the information that would be logically implied by joining them together:

- $v \sqsupset z$ holds

- $e \sqsupset((z \wedge \neg m) \vee(m \wedge \neg z))$ holds

- $v \wedge e \sqsupset(z \wedge((z \wedge \neg m) \vee(m \wedge \neg z)))$ fails

Showing how the Conjunction principle fails is the key point of this section. Before closing a quick look at the Disjunction principle is in order. The failure of the Conjunction principle, as demonstrated above with the zebra-mule example, carries over to the Disjunction principle. Continuing on from that example, given contraposition we have:

\footnotetext{
${ }^{20}$ In its full form this statement is an analytic truth. For our purposes this is a simplified version, since if something is an equid it could also be a horse or donkey. Adding these would not affect the argument though, since all the options are mutually exclusive.
} 
- $v \sqsupset z$ therefore $\neg z \sqsupset \neg v$

- $v \sqsupset((z \wedge \neg m) \vee(m \wedge \neg z))$ therefore $\neg((z \wedge \neg m) \vee(m \wedge \neg z)) \sqsupset \neg v$

Combining these with the Disjunction principle would result in:

- $(\neg z \vee \neg((z \wedge \neg m) \vee(m \wedge \neg z))) \sqsupset \neg v$

- $\neg(z \wedge((z \wedge \neg m) \vee(m \wedge \neg z))) \sqsupset \neg v$

- $v \sqsupset(z \wedge((z \wedge \neg m) \vee(m \wedge \neg z)))$

from which the unwanted $v \sqsupset \neg m$ results as above.

We have employed a system of multi-modal logic in our analysis of relevant alternative approaches to information. Whilst its employment has facilitated the analysis, the logic by itself is insufficient and extra-logical considerations need to be factored in when assessing arguments. The logical properties of information flow endorsed by an account depend on how indexes are assigned to modal operators when assessing arguments. Thus placing constraints on acceptable assignments can be used to determine the properties endorsed by an account.

As we have seen, the contextualist approach works by fixing a context for evaluation and judging the argument (premises and conclusion) against that context. Therefore, the set of valid information flow relations for a contextualist approach can essentially be found in the set of corresponding valid arguments of the mono-modal logic, which in this case was $\mathbf{T}$.

Dretske's account calls for the most 'customisation'. Going by our investigation, out of the seven properties of information flow listed in Section 1.1. Transitivity, Conjunction and Disjunction are the three that do not hold. Thus a suitable core formal definition of $\sqsupset$ for Dretske's account will be such that it invalidates these three properties and validates the others. The following restriction on modal operator indexing is possibly one way to achieve this:

- For all formulas $\mathrm{A}$ and $\mathrm{B}$ and indices $x$ and $y$, if $A \vdash B$ then the argument $\square_{y} A \vdash \square_{x} B$ is to be evaluated only against models that are based on frames with the following accessibility relation property: $w R_{x} w^{\prime} \Rightarrow w R_{y} w^{\prime}$.

Thus going by this extra-logical condition, an argument $\square_{y}(p \supset q \wedge r) \vdash \square_{x}(p \supset q)$ for example will always hold since there can be no countermodel in which it is the case that $w R_{x} w^{\prime}$ but it is not the case that $w R_{y} w^{\prime}$. In terms of Dretske's account, this would suggest that when $A \vdash B$ the relevant alternatives associated with $B$ must form a subset of the relevant alternatives associated with $A$.

Another way to generate a suitable candidate definition of $\sqsupset$ for Dretske's account that gets these validities and invalidities is to define $A \sqsupset B$ as $\square(A \supset B)$ in the monotonic non-normal modal logic characterised by the following rules and axioms ${ }^{21}$

- Propositional Calculus (PC)

\footnotetext{
${ }^{21}$ See Chellas(1980)] for a comprehensive look at such systems of non-normal modal logic.
} 
- The axiom: $\square A \supset A(\mathrm{~T})$

- The rule: $\frac{A}{\square A}(\mathrm{~N})$ [where $A$ is a theorem]

- The rule: $\frac{A \supset B}{\square A \supset \square B}(\mathrm{RM})$ [where $A \supset B$ is a theorem]

On a final note, with the use of this non-normal modal logic, it might seem that a definition such as the following that is based on a high threshold of conditional probability could do the job:

$$
A \sqsupset B=_{d f} \operatorname{Pr}(B \mid A) \geq 0.99
$$

But as the following example shows, such an approach will not work. Going back to the zebramule counter-example to the conjunction principle, we make the following example assignments:

1. $\operatorname{Pr}(z \mid v)=0.99$

2. $\operatorname{Pr}((z \wedge \neg m) \vee(m \wedge \neg z) \mid v)=1$

Statement 1 expresses the approach whereby rather than employing the relevant alternatives strategy and assigning a conditional probability of 1 against a certain set of relevant alternatives, a probabilistic statement below 1 is made instead. The probability that something is a zebra given that it looks like a zebra is very high but not 1 , since there is a minute probability that some skeptical scenario could obtain. Statement 2 simply expresses the fact that the visual signal 'zebra' guarantees that the creature is a zebra or a disguised mule.

But it is a fact of probability that if $\operatorname{Pr}(B \mid A)=x$ and $\operatorname{Pr}(C \mid A)=1$, then $\operatorname{Pr}(B \wedge C \mid A)=x$. Given this setup, the unwanted $v \sqsupset \neg m$ would be a consequence:

- $\operatorname{Pr}(z \wedge((z \wedge \neg m) \vee(m \wedge \neg z)) \mid v)=0.99$

- $\therefore \operatorname{Pr}(\neg m \mid v) \geq 0.99$

Thus whilst the Conjunction and Transitivity principles fail and knowledge is not closed under entailment or conjunction, the failure of these principles/inferences cannot be captured by using a definition of information flow based on a high probability threshold.

The key task of this paper has been to analyse Dretske's account of information flow via its translation into a modal logical framework. A future project, which the results of this paper could help to inform, is the development an epistemic logic based on Dretske's account of knowledge. It goes without saying that the logic behind Dretske's account of information and knowledge will be anything but straightforward and there are certain effects of closure denial that Dretske seems to have not fully appreciated. Whilst this is not a problem in itself (and makes things more fun), explicating this account and its logic is an important project, to which hopefully this paper has made a worthwhile contribution. 


\section{References}

[Adams(2005)] Adams F (2005) Tracking theories of knowledge. Veritas 50:1-35, draft available online at http://revistaseletronicas.pucrs.br/ojs/index.php/veritas/article/ viewFile/1813/1343

[Baumann(2006)] Baumann P (2006) Information, closure, and knowledge: On jager's objection to dretske. Erkenntnis 64:403-408

[Black(2006)] Black T (2006) Contextualism in epistemology. In: Fieser J, Dowden B (eds) Internet Encyclopedia of Philosophy, july 15, 2006 edn, uRL $=<$ http://http://www.iep.utm.edu/ contextu/>

[Chellas(1980)] Chellas BF (1980) Modal Logic: An Introduction. Cambridge University Press

[Cohen and Meskin(2006)] Cohen J, Meskin A (2006) An objective counterfactual theory of information. Australasian Journal of Philosophy 84(3):333-352

[Collins(2006)] Collins JM (2006) Epistemic closure principles. In: Fieser J, Dowden B (eds) Internet Encyclopedia of Philosophy, june 13, 2006 edn, uRL $=\langle$ http://www.iep.utm.edu/ epis-clo/>

[Demir(2011)] Demir H (2011) The counterfactual theory of information revisited. Australasian Journal of Philosophy pp 1-3, online First

[Dretske(1970)] Dretske F (1970) Epistemic operators. Journal of Philosophy 67:1007-1023

[Dretske(1971)] Dretske F (1971) Conclusive reasons. Australasian Journal of Philosophy 49:1-22

[Dretske(1981)] Dretske F (1981) Knowledge and the Flow of Information. MIT Press

[Dretske(1983)] Dretske F (1983) Precis of knowledge and the flow of information. Behavioral and Brain Sciences 6(1):55-63

[Dretske(2003)] Dretske F (2003) Skepticism: What perception teaches. In: Luper S (ed) The Skeptics: Contemporary Essays, Aldershot: Ashgate Publishing, pp 105-118

[Dretske(2004)] Dretske F (2004) Externalism and modest contextualism. Erkenntnis 61:173-186

[Dretske(2005)] Dretske F (2005) The case against closure. In: Steup M, Sosa E (eds) Contemporary Debates in Epistemology, Blackwell

[Dretske(2006)] Dretske F (2006) Information and closure. Erkenntnis 64:409-413

[Heller(1999)] Heller M (1999) Relevant alternatives and closure. Australasian Journal of Philosophy $77(2): 196-208$

[Jager(2004)] Jager C (2004) Skepticism, information, and closure: Dretske's theory of knowledge. Erkenntnis 61:187-201

[Kripke(2011)] Kripke SA (2011) Philosophical Troubles. Collected Papers Vol I. Oxford University Press

[Lehrer(1990)] Lehrer K (1990) Theory of Knowledge. Westview Press

[Loewer(1983)] Loewer B (1983) Information and belief. Behavioral and Brain Sciences 6(1):75-76

[Luper(2010)] Luper S (2010) The epistemic closure principle. In: Zalta EN (ed) The Stanford Encyclopedia of Philosophy, fall 2010 edn, Stanford University, uRL $=<$ http://plato. stanford.edu/archives/fall2010/entries/closure-epistemic/> 
[Priest(2003)] Priest G (2003) An Introduction to Non-Classical Logic. Cambridge University Press

[Rysiew(2011)] Rysiew P (2011) Epistemic contextualism. In: Zalta EN (ed) The Stanford Encyclopedia of Philosophy, winter 2011 edn

[Shackel(2006)] Shackel N (2006) Shutting dretske's door. Erkenntnis 64:393-401

[Steup(2005)] Steup M (2005) Knowledge and skeptcism. In: Steup M, Sosa E (eds) Contemporary Debates in Epistemology, Blackwell

[Stine(1976)] Stine GC (1976) Skepticism, relevant alternatives, and deductive closure. Philosophical Studies 29:249-261

[Wobcke(2000)] Wobcke W (2000) An information-based theory of conditionals. Notre Dame Journal of Formal Logic 41:95-141 